\title{
Viscosity Characterization of the Value Function of an Investment-consumption Problem in Presence of an Illiquid Asset
}

\author{
Salvatore Federico • Paul Gassiat
}

Communicated by Lars Grüne

Received: date / Accepted: date

\begin{abstract}
We study a problem of optimal investment/consumption over an infinite horizon in a market consisting of a liquid and an illiquid asset. The liquid asset is observed and can be traded continuously, while the illiquid one can only be traded and observed at discrete random times corresponding to the jumps of a Poisson process. The problem is a nonstandard mixed discrete/continuous optimal control problem, which we face by the dynamic programming approach. The main goal of the paper is the characterization of the value function as unique viscosity solution of an associated Hamilton-JacobiBellman equation. We then use such result to build a numerical algorithm, allowing one to approximate the value function and so to measure the cost of illiquidity.
\end{abstract}

Keywords Optimal stochastic control · Hamilton-Jacobi-Bellman equation • Viscosity solutions · Comparison principle · Investment-consumption problem • Liquidity risk

MSC 2010 classification 93E20, 35D40, 35B51, 91G80.

S. Federico (corresponding author)

Dipartimento di Economia, Management e Metodi Quantitativi (Università di Milano)

Via Conservatorio 7, 56122, Milano, Italy.

Tel.: +39-02-50321448

E-mail: salvatore.federico@unimi.it

P. Gassiat

Institut für Mathematik, TU Berlin, Strasse des 17. Juni 136, 10623 Berlin, Germany.

E-mail: gassiat@math.tu-berlin.de 


\section{Introduction}

We study a problem of optimal investment/consumption over an infinite horizon in a market consisting of a liquid and an illiquid asset. The liquid asset is observed and can be traded continuously. In the spirit of [1-3], we consider the following illiquidity issue: we assume that the illiquid asset can only be traded and observed at discrete random times corresponding to the jumps of a Poisson process with intensity $\lambda>0$.

Concerning our way of defining illiquidity, we stress that the illiquid issue we have described above is not the only type of illiquidity that real assets experience in practice. By illiquidity one may intend several things, and many other market illiquidity issues have been indeed considered and modeled in the financial and mathematical literature. We can refer, for instance, to the stream of literature devoted to transaction costs (see, e.g., the monography [4]); to the problem of the presence of a large trader (see, e.g., in discrete time [5], and in continuous time [6-8]); to the presence of bid/ask spreads (see, e.g., $[9]$ ); or to a more complex way of modeling the latter kind of illiquidity (see, e.g., $[10,11])$.

As we have said, our way of modeling the illiquid asset has been already proposed by the literature. However, the aforementioned papers [1-3] deal just with one (illiquid) asset. Within this setting, the recent paper [12] introduces, as in our paper, in the market model also a liquid asset correlated with the illiquid one. ${ }^{1}$ However, in [12] it is assumed full information on the state of the illiquid asset, unlike [2] where the illiquid asset can be observed only at the trading dates. Here we consider the point of view of [2], which seems more realistic, and assume that the illiquid asset can be only observed at the trading dates. Another main difference - besides the different methodology - with respect to [12] is that here we consider general utility, so we cannot perform a reduction of variable by homogeneity, which in [12] is allowed by the choice of CRRA utility.

In Section 2, we set the problem as a mixed discrete/continuous stochastic optimal control problem. Such a problem is not standard in the theory of optimal stochastic control. Thus, in Section 3 - following the approach of [2] by means of a specific dynamic programming principle we reduce the control problem between trading times to a continuous time-inhomogeneous problem. Then we state the main result of the paper (Theorem 3.1), providing the characterization of the value function of the reduced problem as unique continuous viscosity solution of the associated Hamilton-Jacobi-Bellman (HJB) equation. This result allows us to perform in Section 4 a numerical iterative scheme to approximate this value function, which is not straightforward due to the presence of a nonlocal term in the HJB equation. In Section 5 we exploit the results obtained providing some first answers to the problem: we describe the struc-

\footnotetext{
1 Also in [13] the market is composed by a liquid and an illiquid asset. However, there the problem is over a finite horizon and the illiquid asset cannot be traded: the wealth held in the illiquid asset enters just in the optimization functional at the terminal date.
} 
ture of the optimal allocation policy in the illiquid asset and give a numerical measure of the cost of illiquidity.

In order to go further into the solution and state the optimal allocation in the liquid asset, as well as the optimal consumption rate, one has to prove regularity results for the value function. These results are the object, in the case of power utility, of the companion paper [14] (see also [15]), where more numerical tests are performed.

\section{Market Model and Optimization Problem}

Let us consider a complete filtered probability space $\left(\Omega, \mathcal{F},\left(\mathcal{F}_{t}\right)_{t \geq 0}, \mathbb{P}\right)$ satisfying the usual conditions, on which there are defined the following objects.

- A Poisson process $\left(N_{t}\right)_{t \geq 0}$, with intensity $\lambda>0$; we denote by $\left(\mathcal{N}_{t}\right)_{t \geq 0}$ the filtration generated by this process and by $\left(\tau_{n}\right)_{n \geq 1}$ its jump times; moreover we set $\tau_{0}:=0$.

- Two independent standard Brownian motions $\left(B_{t}\right)_{t \geq 0}$ and $\left(W_{t}\right)_{t>0}$, independent also of the Poisson process $\left(N_{t}\right)_{t \geq 0}$; we denote by $\left(\mathcal{B}_{t}\right)_{t \geq 0}$ and $\left(\mathcal{W}_{t}\right)_{t \geq 0}$ the filtration generated by $B$ and $W$ respectively.

The market model we consider on this probability space is composed by a riskless asset with constant return rate, which for sake of simplicity we consider equal to 0 , and two risky assets with correlation $\rho \in]-1,1[$

- A liquid risky asset that can be traded continuously; it is described by a stochastic process denoted by $S_{t}^{L}$ whose dynamics is

$$
d S_{t}^{L}=b_{L} S_{t}^{L} d t+\sigma_{L} S_{t}^{L} d W_{t},
$$

where $b_{L} \in \mathbb{R}$ and $\sigma_{L}>0$.

- An illiquid risky asset that can only be traded at the trading times $\tau_{n}$; it is described by a stochastic process denoted by $S_{t}^{I}$, whose dynamics is

$$
d S_{t}^{I}=b_{I} S_{t}^{I} d t+\sigma_{I} S_{t}^{I}\left(\rho d W_{t}+\sqrt{1-\rho^{2}} d B_{t}\right)
$$

where $b_{I} \in \mathbb{R}$ and $\sigma_{I}>0$.

Without any loss of generality we assume $S_{0}^{L}=S_{0}^{I}=1$. We define the $\sigma$ algebra

$$
\mathcal{I}_{t}=\sigma\left(S_{\tau_{n}}^{I} \mathbf{1}_{\left\{\tau_{n} \leq t\right\}}, n \geq 0\right), \quad t \geq 0 .
$$

Moreover, we define

$$
\mathcal{G}_{t}^{0}:=\mathcal{N}_{t} \vee \mathcal{I}_{t} \vee \mathcal{W}_{t}=\sigma\left(\tau_{n}, S_{\tau_{n}}^{I} ; \tau_{n} \leq t\right) \vee \mathcal{W}_{t}, \quad t \geq 0
$$

The observation filtration we consider is

$$
\mathbb{G}=\left(\mathcal{G}_{t}\right)_{t \geq 0} ; \quad \mathcal{G}_{t}:=\mathcal{G}_{t}^{0} \vee \sigma(\mathbb{P} \text {-null sets })
$$


This means that at time $t$ the agent knows the past of the liquid asset up to time $t$, the trading dates of the illiquid assets occurred before $t$, and the values of the illiquid asset at such trading dates.

In the setting above, we define a set of admissible trading/consumption strategies in the following way. Consider all the triplets of processes $\left(c_{t}, \pi_{t}, \alpha_{k}\right)$ such that:

(h1) $c=\left(c_{t}\right)_{t \geq 0}$ is a continuous-time nonnegative process $\left(\mathcal{G}_{t}\right)_{t \geq 0}$-predictable and with locally integrable trajectories; $c_{t}$ represents the consumption rate at time $t$.

(h2) $\pi=\left(\pi_{t}\right)_{t \geq 0}$ is a continuous-time process $\left(\mathcal{G}_{t}\right)_{t \geq 0}$-predictable with locally square integrable trajectories; $\pi_{t}$ represents the amount of money invested in the liquid asset at time $t$.

(h3) $\alpha=\left(\alpha_{k}\right)_{k \in \mathbb{N}}$ is a discrete-time process and $\alpha_{k}$ is $\mathcal{G}_{\tau_{k}}$-measurable for each $k \in \mathbb{N} ; \alpha_{k}$ represents the amount of money invested in the illiquid asset in the interval $\left.] \tau_{k}, \tau_{k+1}\right]$.

Given a triplet $(c, \pi, \alpha)$ satisfying the requirements (h1)-(h3) above and an initial wealth $r \geq 0$, we can consider the process $R_{t}$ representing the wealth associated to such strategy. Its dynamics can be defined by recursion on $k \in \mathbb{N}$ by

$R_{0}=r$
$\left.\left.R_{t}=R_{\tau_{k}}+\int_{\tau_{k}}^{t}\left(-c_{s} d s+\pi_{s}\left(b_{L} d s+\sigma_{L} d W_{s}\right)\right)+\alpha_{k}\left(\frac{S_{t}^{I}}{S_{\tau_{k}}^{I}}-1\right), t \in\right] \tau_{k}, \tau_{k+1}\right]$.

As a class of admissible controls we consider all the triplets of processes $(c, \pi, \alpha)$ satisfying (h1)-(h3) above and such that the corresponding wealth process $R_{t}$ is nonnegative (no-bankruptcy constraint). One can see without big difficulty that this requirement is equivalent to require

$$
\begin{gathered}
0 \leq \alpha_{k} \leq R_{\tau_{k}}, \quad \forall k \geq 0 \\
\int_{\tau_{k}}^{t}\left(\left(c_{s}-b_{L} \pi_{s}\right) d s-\sigma_{L} \pi_{s} d W_{s}\right) \leq R_{\tau_{k}}-\alpha_{k}, \quad \forall t \in\left[\tau_{k}, \tau_{k+1}[.\right.
\end{gathered}
$$

The class of admissible controls depends on the initial wealth $R_{0}=r$. We denote this class by $\mathcal{A}(r)$, noticing that it is nonempty for every $r \geq 0$, as the control $(c, \pi, \alpha) \equiv(0,0,0)$ belongs to $\mathcal{A}(r)$.

Throughout the paper, $\mathbb{R}_{+}$shall denote the set $[0,+\infty[$. Given a utility function $U: \mathbb{R}_{+} \longrightarrow \mathbb{R}$, the optimization problem we want to solve is

$$
\text { Maximize } \mathbb{E}\left[\int_{0}^{\infty} e^{-\beta s} U\left(c_{s}\right) d s\right], \quad \text { over }(c, \pi, \alpha) \in \mathcal{A}(r) .
$$

We make the following assumptions on the utility function $U$ and the discount factor $\beta$. 
Assumption 2.1 $U$ is continuous, nondecreasing, concave and bounded from below (without any loss of generality we assume that $U(0)=0$ ). Moreover, $U$ satisfies the growth condition, for some $K_{U}>0$ and $\left.p \in\right] 0,1[$,

$$
U(c) \leq K_{U} c^{p}
$$

Assumption $2.2 \beta$ is such that

$$
\beta>k_{p}
$$

where

$k_{p}:=\sup _{u_{L} \in \mathbb{R}, u_{I} \in[0,1]}\left\{p\left(u_{L} b_{L}+u_{I} b_{I}\right)-\frac{p(1-p)}{2}\left(u_{L}^{2} \sigma_{L}^{2}+u_{I}^{2} \sigma_{I}^{2}+2 \rho u_{L} u_{I} \sigma_{L} \sigma_{I}\right)\right\}$.

For convenience we set

$$
\tilde{k}_{p}:=\sup _{u_{I} \in[0,1]}\left\{p\left(b_{I}-\frac{\rho b_{L} \sigma_{I}}{\sigma_{L}}\right) u_{I}-\frac{p(1-p)}{2} \sigma_{I}^{2}\left(1-\rho^{2}\right) u_{I}^{2}\right\} .
$$

so that

$$
k_{p}=\frac{p}{2(1-p)} \frac{b_{L}^{2}}{\sigma_{L}^{2}}+\tilde{k}_{p}
$$

Remark 2.1 The assumption on $\beta$ is related to the investment/consumption problem with the same assets but in a liquid market. Let $p \in] 0,1[$ and consider an agent with initial wealth $r$, consuming at rate $c_{t}$ and investing in $S_{t}^{L}$ and $S_{t}^{I}$ continuously with respective proportions $u_{t}^{S^{L}}$ and $u_{t}^{S^{I}}$ and under the constraint that $u_{t}^{S^{I}} \in[0,1]$. Suppose, moreover, that the preferences of the agent are represented by the utility function $U^{(p)}(c)=c^{p} / p$. Let us denote by $\mathcal{A}_{\text {Mert }}(r)$ the set of strategies keeping the wealth nonnegative and define the value function

$$
V_{M e r t}^{(p)}(r)=\sup _{\left(u^{L}, u^{I}, c\right) \in \mathcal{A}_{M e r t}(r)} \mathbb{E}\left[\int_{0}^{\infty} e^{-\beta t} U^{(p)}\left(c_{t}\right) d t\right] .
$$

This is a constrained Merton problem which dominates our problem, in the sense that $V_{M e r t}^{(p)}(r)$ is higher of the optimal value of our problem, up to the multiplicative constant $K_{U}$ of (2). One can see (for instance by solving the HJB equation) that $V_{\text {Mert }}^{(p)}$ is finite if and only if (3) is satisfied and that in this case

$$
V_{\text {Mert }}^{(p)}(r)=\left(\frac{1-p}{\beta-k_{p}}\right)^{1-p} r^{p}
$$

Therefore, condition (3) guarantees together with (2) the finiteness for our problem too. 
Remark 2.2 It is worth to stress that the assets $S^{L}, S^{I}$, as well as the wealth $R$, have continuous dynamics (no jumps). What is jumping in our model is the information, which is updated each time that an investment opportunity occurs, i.e. at the dates $\tau_{k}$ that are the jump times of the Poisson process $N$, which enters into the model only to define the random trading times $\tau_{k}$. One could be interested also to another kind of optimization problem, when is the stock to have jumps. For this kind of optimal control problems we can refer, e.g., to the monography [16] and the references therein.

\section{Dynamic Programming and HJB Equation}

Let us denote by $V$ the value function of the stochastic control problem (1):

$$
V(r)=\sup _{(c, \pi, \alpha) \in \mathcal{A}(r)} \mathbb{E}\left[\int_{0}^{\infty} e^{-\beta s} U\left(c_{s}\right) d s\right], \quad r \geq 0 .
$$

Proposition 3.1 $V$ is everywhere finite, concave, p-Hölder continuous and nondecreasing. Moreover

$$
V(r) \leq K_{V} r^{p}, \quad \text { for some } K_{V}>0
$$

Proof. As we have already observed in Remark 2.1, finiteness and (6) follow from (2) and (3), by comparing with a constrained Merton problem.

Concavity of $V$ comes from concavity of $U$ and linearity of the state equation by standards arguments; also monotonicity is consequence of standard arguments due to monotonicity of $U$ (detailed proofs of such properties for a very similar problem can be found in Section 2.4 in [17]; the same arguments apply to our problem). Finally, $p$-Hölder continuity follows from concavity and monotonicity of $V$, and from (6).

Following [2], we state a suitable Dynamic Programming Principle (DPP) to reduce our mixed discrete/continuous problem to a standard one between two trading times. Note that due to the mixed discrete/continuous nature of the control problem (both for the filtration and for the state processes), this result is nonstandard and not covered by the usual references.

Proposition 3.2 (Dynamic Programming Principle) For every $r \geq 0$ we have the following equality:

$$
V(r)=\sup _{(c, \pi, \alpha) \in \mathcal{A}(r)} \mathbb{E}\left[\int_{0}^{\tau_{1}} e^{-\beta s} U\left(c_{s}\right) d s+e^{-\beta \tau_{1}} V\left(R_{\tau_{1}}\right)\right] .
$$

Proof. The proof can be obtained by the same procedure as in [18], and for brevity we only indicate where it must be modified. The main difference comes from the fact that, unlike in [18], there is some additional information between $\tau_{n}$ and $\tau_{n+1}$ brought by $\left(\mathcal{W}_{t}\right)$, so that all the processes considered are no longer deterministic on $\left.] \tau_{n}, \tau_{n+1}\right]$ given $\mathcal{G}_{\tau_{n}}$, but only predictable with respect to $\left(\mathcal{W}_{t}\right)$ 
in $\left.] \tau_{n}, \tau_{n+1}\right]$. More precisely, one should use the fact that a process $\left(\xi_{t}\right)_{t \geq 0}$ is $\mathbb{G}$-predictable if and only if it admits a decomposition

$$
\xi_{t}(\omega)=f_{0}(s, \omega) \mathbf{1}_{\left\{t \leq \tau_{1}\right\}}+\sum_{n \geq 1} f_{n}\left(s, \omega, \tau_{1}, I_{\tau_{1}}, \ldots, \tau_{n}, I_{\tau_{n}}\right) \mathbf{1}_{\left\{\tau_{n}<t \leq \tau_{n+1}\right\}} \text { a.s. }
$$

(see e.g. Lemma 2.1 in [19]), where each $f_{n}$ is $\mathcal{P}^{W} \otimes \mathcal{B}\left(\mathbb{R}^{2 n}\right)$-measurable, $\mathcal{P}^{W}$ being the predictable $\sigma$-algebra corresponding to $\left(\mathcal{W}_{t}\right)$. One then proceeds exactly as in [18] by considering conditional controls and using countable selection (one needs in addition a technical result similar to Lemma 3.2 in [20] for the shifting procedure).

We can use this DPP to relate our original problem to a standard continuoustime control problem. First of all, let $\mathcal{M}(\mathcal{S} ; \mathbb{R})$ denote the space of measurable functions from $\mathcal{S} \subset \mathbb{R}_{+}^{n}$ to $\mathbb{R}$, and let us define the linear operator

$$
\begin{array}{clc}
G: \mathcal{M}\left(\mathbb{R}_{+} ; \mathbb{R}\right) & \longrightarrow & \mathcal{M}\left(\mathbb{R}_{+}^{3} ; \mathbb{R}\right) \\
\psi & \longmapsto G[\psi](t, x, y):=\mathbb{E}\left[\psi\left(x+y J_{t}\right)\right],
\end{array}
$$

where

$$
\frac{d J_{t}}{J_{t}}=\left(b_{I}-\rho b_{L} \frac{\sigma_{I}}{\sigma_{L}}\right) d t+\sigma_{I} \sqrt{1-\rho^{2}} d B_{t}, \quad J_{0}=1 .
$$

For each $x \geq 0$ and $t \geq 0$, let $\mathcal{A}_{t}(x)$ be the set of couples of stochastic processes $\left(c_{s}, \pi_{s}\right)_{s \geq t}$ such that

- $\left(c_{s}\right)_{s \geq t}$ is $\left(\mathcal{W}_{s}\right)_{s \geq t}$-predictable, nonnegative and has locally integrable trajectories;

- $\left(\pi_{s}\right)_{s \geq t}$ is $\left(\mathcal{W}_{s}\right)_{s \geq t}$-predictable and has locally square integrable trajectories;

- $x+\int_{t}^{T}\left(-c_{s} d s+\pi_{s}\left(b_{L} d s+\sigma_{L} d W_{s}\right)\right) \geq 0$, for all $T \geq t$.

Define

$$
\widehat{V}(t, x, y):=\sup _{(c, \pi) \in \mathcal{A}_{t}(x)} \mathcal{J}(t, x, y ; c, \pi), \quad(t, x, y) \in \mathbb{R}_{+}^{3},
$$

where

$$
\begin{aligned}
& \mathcal{J}(t, x, y ; c, \pi) \\
& \quad=\mathbb{E} \int_{t}^{\infty} e^{-(\beta+\lambda)(s-t)}\left(U\left(c_{s}\right)+\lambda G[V]\left(s, X_{s}^{t, x, \pi, c}, Y_{s}^{t, y}\right)\right) d s,
\end{aligned}
$$

with $\left(X_{s}^{t, x, c, \pi}\right)_{s \geq t},\left(Y_{s}^{t, y}\right)_{s \geq t}$ solutions to the SDEs

$$
\begin{aligned}
& d X_{s}=-c_{s} d s+\pi_{s}\left(b_{L} d s+\sigma_{L} d W_{s}\right), \quad X_{t}^{t, x, c, \pi}=x, \\
& d Y_{s}=\rho Y_{s}\left(\frac{b_{L} \sigma_{I}}{\sigma_{L}} d t+\sigma_{I} d W_{s}\right), \quad Y_{t}^{t, y}=y .
\end{aligned}
$$


We notice that the problem of optimizing the functional above is not autonomous due to the dependence of $G[V]$ on time. Now, given $(c, \pi, \alpha) \in \mathcal{A}(r)$, one has

$$
R_{t}=X_{t}^{0, r-\alpha_{0}, c, \pi}+J_{t} Y_{t}^{0, \alpha_{0}}, \quad \forall t \leq \tau_{1}
$$

So, taking into account that $\tau_{1}$ is independent of $\mathcal{W}_{\infty} \vee \mathcal{B}_{\infty}$ with exponential law of parameter $\lambda$, that $J$ is $\mathcal{B}_{\infty}$-measurable and independent of $\mathcal{W}_{\infty}$, and that $c, X^{0, r-\alpha_{0}, c, \pi}, Y^{0, \alpha_{0}}$ are $\left(\mathcal{W}_{t}\right)$-predictable in $\left[0, \tau_{1}[\right.$, we have, using also Fubini's Theorem,

$$
\begin{aligned}
& \mathbb{E}\left[\int_{0}^{\tau_{1}} e^{-\beta s} U\left(c_{s}\right) d s+e^{-\beta \tau_{1}} V\left(R_{\tau_{1}}\right) \mid \mathcal{W}_{\infty}\right] \\
&=\mathbb{E}\left[\mathbb{E}\left[\int_{0}^{\tau_{1}} e^{-\beta s} U\left(c_{s}\right) d s+e^{-\beta \tau_{1}} V\left(R_{\tau_{1}}\right) \mid \mathcal{W}_{\infty} \vee \mathcal{B}_{\infty}\right] \mid \mathcal{W}_{\infty}\right] \\
&=\mathbb{E}\left[\mathbb{E}\left[\int_{0}^{\infty} \lambda e^{-\lambda t} \int_{0}^{t} e^{-\beta s} U\left(c_{s}\right) d s \mid \mathcal{W}_{\infty} \vee \mathcal{B}_{\infty}\right] \mid \mathcal{W}_{\infty}\right] \\
&+\mathbb{E}\left[\mathbb{E}\left[\int_{0}^{\infty} \lambda e^{-\lambda t} e^{-\beta t} V\left(X_{t}^{0, r-\alpha_{0}, c, \pi}+J_{t} \cdot Y_{t}^{0, \alpha_{0}}\right) d t \mid \mathcal{W}_{\infty} \vee \mathcal{B}_{\infty}\right] \mid \mathcal{W}_{\infty}\right] \\
&= \mathbb{E}\left[\int_{0}^{\infty} e^{-\beta s} U\left(c_{s}\right) \int_{s}^{\infty} \lambda e^{-\lambda t} d t d s \mid \mathcal{W}_{\infty}\right] \\
&+\mathbb{E}\left[\int_{0}^{\infty} \lambda e^{-(\lambda+\beta) t} G[V]\left(t, X_{t}^{0, r-\alpha_{0}, c, \pi}, Y_{t}^{0, \alpha_{0}}\right) d t \mid \mathcal{W}_{\infty}\right] \\
&= \int_{0}^{\infty} e^{-(\beta+\lambda) t}\left(U\left(c_{t}\right)+\lambda G[V]\left(t, X_{t}^{0, r-\alpha_{0}, c, \pi}, Y_{t}^{0, \alpha_{0}}\right)\right) d t
\end{aligned}
$$

Taking the expectation in the equality above and using (7) we get

$$
V(r)=\sup _{0 \leq a \leq r} \widehat{V}(0, r-a, a), \quad r \geq 0
$$

Associating to every locally bounded function $\hat{v}: \mathbb{R}_{+}^{3} \rightarrow \mathbb{R}$ the function

$$
\mathcal{H} \hat{v}: \mathbb{R}_{+} \rightarrow \mathbb{R}, \quad[\mathcal{H} \hat{v}](r):=\sup _{0 \leq a \leq r} \hat{v}(0, r-a, a)
$$

by the arguments above we can rewrite the original problem as

$$
V(r)=[\mathcal{H} \widehat{V}](r)
$$

The problems (10) and (15) are coupled in the sense that $\widehat{V}$ is expressed in terms of $V$ in (10) and, viceversa, $V$ can be expressed in terms of $\widehat{V}$ by (15). 
3.1 Properties of $\widehat{V}$

In this subsection we prove some qualitative properties of the value function $\widehat{V}$. First, we start by studying some properties of the operator $G$.

Proposition 3.3 We have the following statements regarding the operator $G$.

(i) $G$ is well defined on the set of measurable functions with polynomial growth.

(ii) $G$ is positive, in the sense that it maps positive functions into positive ones.

(iii) $G$ maps increasing functions to functions which are increasing with respect to both $x$ and $y$.

(iv) $G$ maps concave functions to functions which are concave with respect to $(x, y)$.

(v) If $\left.\psi(r)=r^{p}, p \in\right] 0,1[$, then

$$
0 \leq G[\psi](t, x, y) \leq e^{\tilde{k}_{p} t}(x+y)^{p}, \quad \forall t \geq 0, \forall(x, y) \in \mathbb{R}_{+}^{2} .
$$

(vi) Let $p \in] 0,1]$ and $\psi$ a p-Hölder continuous function. Then there exists some constant $C \geq 0$ such that for all $t \geq 0, x, x^{\prime}, y, y^{\prime} \geq 0$, and $0<h \leq 1$,

$$
\begin{aligned}
\left|G[\psi](t, x, y)-G[\psi]\left(t, x^{\prime}, y\right)\right| & \leq C\left|x-x^{\prime}\right|^{p}, \\
\left|G[\psi](t, x, y)-G[\psi]\left(t, x, y^{\prime}\right)\right| & \leq C e^{\tilde{k}_{p} t}\left|y-y^{\prime}\right|^{p}, \\
|G[\psi](t, x, y)-G[\psi](t+h, x, y)| & \leq C e^{\tilde{k}_{p} t} y^{p} h^{p / 2} .
\end{aligned}
$$

Proof. (i)-(iv) are straightforward.

(v). If $x=y=0$ the claim is obvious, so we assume $x+y>0$. By a straightforward application of Itô's formula and the definition of $\tilde{k}_{p}$, we see that the process

$$
\left(e^{-\tilde{k}_{p} t}\left(x+y J_{t}\right)^{p}\right)_{t \geq 0}
$$

is a supermartingale, which implies (16).

(vi). (17) is obvious, and (18) follows directly from (v). To prove (19), fix $(t, x, y) \in \mathbb{R}_{+}^{3}$ and $h \in(0,1]$. Taking into account that $J_{t+h} / J_{t}$ has the same distribution as $J_{h}$ and is independent of $J_{t}$, we can write for some $C>0$

$$
\begin{aligned}
|G[\psi](t, x, y)-G[\psi](t+h, x, y)| & \leq C y^{p} \mathbb{E}\left[\left|J_{t}-J_{t+h}\right|^{p}\right] \\
& =C y^{p} \mathbb{E}\left[\left|J_{t}\right|^{p}\left|1-\frac{J_{t+h}}{J_{t}}\right|^{p}\right] \\
& =C y^{p} \mathbb{E}\left[\left|J_{t}\right|^{p}\right] \mathbb{E}\left[\left|1-J_{h}\right|^{p}\right] \\
& \leq C e^{\tilde{k}_{p} t} y^{p} \mathbb{E}\left[\left|1-J_{h}\right|^{p}\right] .
\end{aligned}
$$

We have $J_{h}=e^{\alpha h+\beta \sqrt{h} N}$, where $\alpha, \beta$ are constants and where $N \sim \mathcal{N}(0,1)$. Since $\left|e^{\xi}-1\right| \leq|\xi|\left(e^{\xi}+1\right)$ for all $\xi \in \mathbb{R}$, we obtain for some $C_{1}>0$

$$
\left.\left.\frac{\mathbb{E}\left[\left|1-J_{h}\right|^{p}\right]}{h^{p / 2}} \leq \mathbb{E}\left[(\alpha \sqrt{h}+\beta N)^{p}\left(e^{\alpha h+\beta \sqrt{h} N}+1\right)^{p}\right] \leq C_{1}, \quad \forall h \in\right] 0,1\right] .
$$

The claim follows combining (21) with (20). 
Lemma 3.1 For $\left.(t, x, y) \in \mathbb{R}_{+}^{3},(c, \pi) \in \mathcal{A}_{t}(x), p \in\right] 0,1[$,

$$
\mathbb{E}\left[\left(X_{s}^{t, x, c, \pi}+Y_{s}^{t, y}\right)^{p}\right] \leq e^{\frac{p}{1-p} \frac{b_{L}^{2}}{2 \sigma_{L}^{2}}(s-t)}(x+y)^{p}, \quad \forall s \geq t .
$$

Proof. Fix $(t, x, y) \in \mathbb{R}_{+}^{3}$ and $(c, \pi) \in \mathcal{A}_{t}(x)$. First of all we notice that by standard comparison of SDE's we have

$$
X^{t, x, c, \pi} \leq X^{t, x, 0, \pi} .
$$

On the other hand we have

$$
\frac{d\left(X_{s}^{t, x, 0, \pi}+Y_{s}^{t, y}\right)}{X_{s}^{t, x, 0, \pi}+Y_{s}^{t, y}}=U_{s}\left(b_{L} d s+\sigma_{L} d W_{s}\right)
$$

where

$$
U_{s}=\frac{\pi_{s}+\rho \frac{\sigma_{I}}{\sigma_{L}} Y_{s}^{t, y}}{X_{s}^{t, x, 0, \pi}+Y_{s}^{t, y}}
$$

Noticing that

$$
\frac{p}{1-p} \frac{b_{L}^{2}}{2 \sigma_{L}^{2}}=\sup _{u \in \mathbb{R}}\left\{p b_{L} u-\frac{p(1-p)}{2} \sigma_{L}^{2} u^{2}\right\}
$$

it is then a straightforward application of Itô's formula to check that the process

$$
\left(e^{-\frac{p}{1-p} \frac{b_{L}^{2}}{2 \sigma_{L}^{2}}(s-t)}\left(X_{s}^{t, x, 0, \pi}+Y_{s}^{t, y}\right)^{p}\right)_{s \geq t}
$$

is a local supermartingale, and, being nonnegative, a true supermartingale. Therefore, we have the claim for $c=0$. Then, the general claim follows from (23).

Proposition $3.4 \widehat{V}(t, \cdot)$ is concave with repect to $(x, y)$ and nondecreasing with respect to $x$ and $y$ for every $t \geq 0$. Moreover it satisfies the boundary condition

$$
\widehat{V}(t, 0, y)=\mathbb{E} \int_{t}^{\infty} e^{-(\beta+\lambda)(s-t)} \lambda G[V]\left(s, 0, Y_{s}^{t, y}\right) d s, \quad \forall t \geq 0, \forall y \geq 0 .
$$

In particular, since by Assumption 2.1 it is $U(0)=0$, due to Proposition $3.3(v)$ we have

$$
\widehat{V}(t, 0,0)=0, \quad \forall t \geq 0
$$

Finally, $\widehat{V}$ is continuous on $\mathbb{R}_{+}^{3}$, and satisfies for some $K_{\widehat{V}}>0$ the growth condition

$$
0 \leq \widehat{V}(t, x, y) \leq K_{\widehat{V}} e^{\tilde{k}_{p} t}(x+y)^{p}, \quad \forall(t, x, y) \in \mathbb{R}_{+}^{3} .
$$


Proof. Concavity and monotonicity. Since $V$ is concave and nondecreasing, by Proposition 3.3(iii, iv), $G[V](t, \cdot)$ is concave in $(x, y)$ and nondecreasing in $x, y$ on $\mathbb{R}_{+}^{2}$. Then concavity and monotonicity of $\widehat{V}$ follow by standard arguments, considering also the linearity of the SDE's (12)-(13).

Boundary condition. Equality $(24)$ is due to the fact that $\mathcal{A}_{t}(0)=\{(0,0)\}$, so

$$
\widehat{V}(t, 0, y)=\mathcal{J}(t, 0, y ; 0,0)=\mathbb{E} \int_{t}^{\infty} e^{-(\beta+\lambda)(s-t)} \lambda G[V]\left(s, 0, Y_{s}^{t, y}\right) d s .
$$

Continuity. We prove the continuity of $\widehat{V}$ in several steps.

1) Continuity of $\widehat{V}(t, \cdot)$ in $(0,+\infty) \times(0,+\infty)$ follows from concavity.

2) Here we prove the continuity of $\widehat{V}(t, \cdot, y)$ at $x=0^{+}$. First of all we note that (24) holds at $x=0$. Therefore, using the monotonicity of $V$ and Proposition 3.3 (iii), we get

$$
0 \leq \mathcal{J}(t, x, y ; 0,0)-\mathcal{J}(t, 0, y ; 0,0) \leq \widehat{V}(t, x, y)-\widehat{V}(t, 0, y) .
$$

On the other hand, using the Hölder continuity of $V$ and (17), we have for some $K>0$ and all $(c, \pi) \in \mathcal{A}_{t}(x)$

$$
\begin{gathered}
\mathcal{J}(t, x, y ; c, \pi)-\widehat{V}(t, 0, y) \\
\leq \mathbb{E}\left[\int_{t}^{\infty} e^{-(\beta+\lambda)(s-t)}\left\{U\left(c_{s}\right)+\lambda\left|G[V]\left(s, X_{s}^{t, x, c, \pi}, Y_{s}^{t, y}\right)-G[V]\left(s, 0, Y_{s}^{t, y}\right)\right|\right\} d s\right] \\
\leq \mathbb{E}\left[\int_{t}^{\infty} e^{-(\beta+\lambda)(s-t)}\left(U\left(c_{s}\right)+K\left|X_{s}^{t, x, c, \pi}\right|^{p}\right) d s\right] .
\end{gathered}
$$

Taking the supremum over $(c, \pi) \in \mathcal{A}_{t}(x)$ and combining with $(27)$ we get

$$
\begin{aligned}
0 & \leq \widehat{V}(t, x, y)-\widehat{V}(t, 0, y) \\
& \leq \sup _{(c, \pi) \in \mathcal{A}_{t}(x)} \mathbb{E}\left[\int_{t}^{\infty} e^{-(\beta+\lambda)(s-t)}\left(U\left(c_{s}\right)+K\left|X_{s}^{t, x, c, \pi}\right|^{p}\right) d s\right]
\end{aligned}
$$

We want now to estimate the right handside of (28). By definition of $\mathcal{A}_{t}(x)$, we have

$$
0 \leq X_{s}^{t, x, c, \pi}=x+\int_{t}^{s} \pi_{u} \frac{d S_{u}^{L}}{S_{u}^{L}}-\int_{t}^{s} c_{u} d u .
$$

Denoting by $\mathbb{Q}^{L}$ the probability with density process

$$
Z_{t}=\exp \left(-\frac{b_{L}^{2}}{2 \sigma_{L}^{2}} t-\frac{b_{L}}{\sigma_{L}} W_{t}\right)
$$

we have that $L$ is a $\mathbb{Q}^{L}$-martingale. The process $X^{t, x, c, \pi}$ is then a $\mathbb{Q}^{L}$-local supermartingale and, being bounded from below, it is a true $\mathbb{Q}^{L}$-supermartingale. 
Hence, we have $\mathbb{E}\left[Z_{s} X_{s}^{t, x, c, \pi}\right] \leq x$. Now, writing $\left|X_{s}^{t, x, c, \pi}\right|^{p}=\left|Z_{s} X_{s}^{t, x, c, \pi}\right|^{p} Z_{s}^{-p}$, by Hölder's inequality we get

$$
\begin{aligned}
\mathbb{E}\left[\left|X_{s}^{t, x, c, \pi}\right|^{p}\right] & \leq \mathbb{E}\left[Z_{s} X_{s}^{t, x, c, \pi}\right]^{p} \mathbb{E}\left[Z_{s}^{-\frac{p}{1-p}}\right]^{1-p} \\
& \leq x^{p} \exp \left(\left(\frac{p}{1-p} \frac{b_{L}^{2}}{2 \sigma_{L}^{2}}\right) s\right) .
\end{aligned}
$$

Note also that, since $\int_{t}^{\infty} e^{-(\beta+\lambda)(s-t)} U\left(c_{s}\right) d s$ is the utility obtained by the agent trading only in $L$, we have by $(6)$

$$
\sup _{(c, \pi) \in \mathcal{A}_{t}(x)} \mathbb{E}\left[\int_{t}^{\infty} e^{-(\beta+\lambda)(s-t)} U\left(c_{s}\right) d s\right] \leq V(x) \leq K_{V} x^{p} .
$$

Combining (28), (30), (31), and using (3), we get for some $K>0$

$$
0 \leq \widehat{V}(t, x, y)-\widehat{V}(t, 0, y) \leq K x^{p}
$$

and we conclude.

3) Here we prove the continuity of $\widehat{V}(t, x, \cdot)$ at $y=0^{+}$. Using the monotonicity of $V$ and Proposition 3.3(iii) we get

$$
0 \leq \mathcal{J}(t, x, y ; c, \pi)-\mathcal{J}(t, x, 0 ; c, \pi)
$$

On the other hand, using the Hölder continuity of $V,(18)$ and (22), we have for some $K>0$ and for all $(c, \pi) \in \mathcal{A}_{t}(x)$

$$
\begin{aligned}
\mathcal{J}(t, x, y ; c, \pi)-\mathcal{J}(t, x, 0 ; c, \pi) & \leq K e^{\tilde{k}_{p} t} \int_{t}^{\infty} e^{-\left(\beta+\lambda-\tilde{k}_{p}\right)(s-t)} \lambda \mathbb{E}\left[\left(Y_{s}^{t, y}\right)^{p}\right] d s \\
& \leq K e^{\tilde{k}_{p} t} y^{p} \int_{t}^{\infty} e^{-\left(\beta+\lambda-k_{p}\right)(s-t)} \lambda d s \\
& =K \frac{\lambda}{\beta+\lambda-k_{p}} e^{\tilde{k}_{p} t} y^{p} .
\end{aligned}
$$

Therefore, taking the supremum over $(c, \pi) \in \mathcal{A}_{t}(x)$ in (34) and combining with (33), we get

$$
0 \leq \widehat{V}(t, x, y)-\widehat{V}(t, x, 0) \leq \frac{K \lambda}{\beta+\lambda-k_{p}} e^{\tilde{k}_{p} t} y^{p},
$$

and we conclude.

4) Since (35) and (32) are uniform estimates in $x, y$ respectively, combining with the continuity on the lines provided by items 2 ) and 3 ), we get the joint continuity of $\widehat{V}$ w.r.t. $(x, y)$ at the boundary $\left\{(x, y) \in \mathbb{R}_{+}^{2} \mid x=0\right.$ or $\left.y=0\right\}$.

$5)$ Here we prove $p / 2$-Hölder continuity of $\widehat{V}(\cdot, x, y)$. Let $t, t^{\prime} \geq 0$ and suppose that $t^{\prime}=t+h$ for some $0<h \leq 1$. One can associate to each $\left(c_{s}^{t}, \pi_{s}^{t}\right)_{s \geq t} \in \mathcal{A}_{t}(x)$ a control $\left(c_{s}^{t^{\prime}}, \pi_{s}^{t^{\prime}}\right)_{s \geq t^{\prime}} \in \mathcal{A}_{t^{\prime}}(x)$ with the same law and 
viceversa (see Ch.1, Th. 2.10, in [20]). Given that and considering (19) and (22), we have for some $K>0$

$$
\begin{aligned}
& \left|\mathcal{J}\left(t, x, y, c^{t}, \pi^{t}\right)-\mathcal{J}\left(t^{\prime}, x, y, c^{t^{\prime}}, \pi^{t^{\prime}}\right)\right| \\
\leq & \mathbb{E} \int_{t}^{\infty} e^{-(\beta+\lambda)(s-t)} \lambda\left|G[V]\left(s, X^{t, x, c^{t}, \pi^{t}}, Y_{s}^{t, y}\right) d s-G[V]\left(s+h, X^{t, x, c^{t}, \pi^{t}}, Y_{s}^{t, y}\right)\right| d s \\
\leq & K \mathbb{E} \int_{t}^{\infty} e^{-(\beta+\lambda)(s-t)} \lambda h^{p / 2} e^{\tilde{k}_{p} s}\left|Y_{s}^{t, y}\right|^{p} d s \\
\leq & K \frac{\lambda}{\beta+\lambda-k_{p}} e^{\tilde{k}_{p} t} y^{p} h^{p / 2} .
\end{aligned}
$$

Then, taking $\left(c_{s}^{t, \varepsilon}, \pi_{s}^{t, \varepsilon}\right)_{s \geq t} \in \mathcal{A}_{t}(x)$ an $\varepsilon$-optimal control for $(t, x, y)$, and letting $\left(c_{s}^{t^{\prime}, \varepsilon}, \pi_{s}^{t^{\prime}, \varepsilon}\right)_{s \geq t} \in \mathcal{A}_{t^{\prime}}(x)$ be the control associated to it as indicated above, we get

$$
\begin{aligned}
V(t, x, y)-\widehat{V}(t+h, x, y)-\varepsilon & \leq \mathcal{J}\left(t, x, y, c^{t, \varepsilon}, \pi^{t, \varepsilon}\right)-\mathcal{J}\left(t^{\prime}, x, y, c^{t^{\prime}, \varepsilon}, \pi^{t^{\prime}, \varepsilon}\right) \\
& \leq K \frac{\lambda}{\beta+\lambda-k_{p}} e^{\tilde{k}_{p} t} y^{p} h^{p / 2}
\end{aligned}
$$

so, by arbitrariness of $\varepsilon$,

$$
V(t, x, y)-\widehat{V}(t+h, x, y)-\varepsilon \leq K \frac{\lambda}{\beta+\lambda-k_{p}} e^{\tilde{k}_{p} t} y^{p} h^{p / 2},
$$

Reverting the argument we get the opposite inequality, so we conclude that

$$
|\widehat{V}(t, x, y)-\widehat{V}(t+h, x, y)| \leq K \frac{\lambda}{\beta+\lambda-k_{p}} e^{\tilde{k}_{p} t} e^{\tilde{k}_{p} t} y^{p} h^{p / 2}
$$

Hence $\widehat{V}$ is locally $p / 2$-Hölder with respect to $t$.

6) Putting together all the information collected we finally get the continuity of $V$ on $\mathbb{R}_{+}^{3}$.

Growth condition. (26) is obtained by combining (32), (35) and (25).

\subsection{HJB Equation: Viscosity Characterization of $\widehat{V}$}

Let us introduce the notations for vectors of $\mathbb{R}^{2}$ and matrices of $\mathbb{R}^{2 \times 2}$

$$
\mathbf{q}=\left(q_{1}, q_{2}\right), \quad \mathbf{Q}=\left(\begin{array}{ll}
Q_{11} & Q_{12} \\
Q_{21} & Q_{22}
\end{array}\right)
$$

and let $\mathcal{S}_{2}$ denote the space of symmetric $2 \times 2$ matrices. By standard arguments of stochastic control (see, e.g., Ch. 4 in [20]), we can associate to $\widehat{V}$ an HJB equation, which in this case reads as

$$
-\hat{v}_{t}+(\beta+\lambda) \hat{v}-\lambda G[V]-\sup _{c \geq 0, \pi \in \mathbb{R}} H_{c v}\left(y, D_{(x, y)} \hat{v}, D_{(x, y)}^{2} \hat{v} ; c, \pi\right)=0,
$$


where for $(y, \mathbf{q}, \mathbf{Q}) \in \mathbb{R}_{+} \times \mathbb{R}^{2} \times \mathcal{S}_{2}, c \geq 0, \pi \in \mathbb{R}$, the function $H_{c v}$ is

$$
\begin{aligned}
& H_{c v}(y, \mathbf{q}, \mathbf{Q} ; c, \pi) \\
:= & U(c)+\left(\pi b_{L}-c\right) q_{1}+\frac{\rho b_{L} \sigma_{I}}{\sigma_{L}} y q_{2}+\frac{\sigma_{L}^{2} \pi^{2}}{2} Q_{11}+\pi \rho \sigma_{I} \sigma_{L} y Q_{12}+\frac{\rho^{2} \sigma_{I}^{2}}{2} y^{2} Q_{22} .
\end{aligned}
$$

Note that $\sup _{c \geq 0, \pi \in \mathbb{R}} H_{c v}(y, \mathbf{q}, \mathbf{Q} ; c, \pi)$ is finite if $q_{1}>0, Q_{11}<0$, in which case we have

$$
\begin{aligned}
\sup _{c \geq 0, \pi \in \mathbb{R}} H_{c v} & (y, \mathbf{q}, \mathbf{Q} ; c, \pi) \\
& =\widetilde{U}\left(q_{1}\right)-\frac{\left(b_{L} q_{1}+\rho \sigma_{L} \sigma_{I} y Q_{12}\right)^{2}}{2 \sigma_{L}^{2} Q_{11}}+\frac{\rho b_{L} \sigma_{I}}{\sigma_{L}} y q_{2}+\frac{\rho^{2} \sigma_{I}^{2}}{2} y^{2} Q_{22},
\end{aligned}
$$

where $\widetilde{U}$ is the sup-Legendre transform of $U$ on $] 0,+\infty[$, i.e.

$$
\widetilde{U}\left(q_{1}\right):=\sup _{c \geq 0}\left\{U(c)-c q_{1}\right\}, \quad q_{1}>0 .
$$

The term $G[V]$ in (37) is related to $\widehat{V}$ by the coupling relation (15). If we want to decouple the problem and work only with $\widehat{V}$, we must replace $G[V]$ by $G[\mathcal{H} \widehat{V}]$. Correspondingly, the HJB equation becomes

$$
-\hat{v}_{t}+(\beta+\lambda) \hat{v}-\lambda G[\mathcal{H} \hat{v}]-\sup _{c \geq 0, \pi \in \mathbb{R}} H_{c v}\left(y, D_{(x, y)} \hat{v}, D_{(x, y)}^{2} \hat{v} ; c, \pi\right)=0 .
$$

In this way we have decoupled the problem, but the price to pay is now the presence of the nonlocal term $G[\mathcal{H} \hat{v}]$. We work with the latter equation.

Let us denote by $X$ vectors in $\mathbb{R}_{+}^{n}$. We are going to prove that $\widehat{V}$ is the unique constrained viscosity solution to (38) according to the following definition.

Definition 3.1 (1) Given $\varphi \in C\left(\mathbb{R}_{+}^{3} ; \mathbb{R}\right)$, the parabolic superjet of $\varphi$ at $(t, \mathbf{x})$ $\in \mathbb{R}_{+}^{3}$ is defined by:

$$
\begin{aligned}
& \mathcal{P}^{1,2,+} \varphi(t, \mathbf{x}):=\left\{(r, \mathbf{q}, \mathbf{Q}) \in \mathbb{R} \times \mathbb{R}^{2} \times \mathcal{S}_{2}\right. \text { such that } \\
& \varphi\left(s, \mathbf{x}^{\prime}\right) \leq \varphi(t, \mathbf{x})+r(s-t)+\left\langle\mathbf{q}, \mathbf{x}-\mathbf{x}^{\prime}\right\rangle \\
&\left.+\frac{1}{2}\left\langle\mathbf{Q}\left(\mathbf{x}^{\prime}-\mathbf{x}\right), \mathbf{x}^{\prime}-\mathbf{x}\right\rangle+o\left(|s-t|+\left|\mathbf{x}^{\prime}-\mathbf{x}\right|^{2}\right)\right\}
\end{aligned}
$$

We define its closure $\overline{\mathcal{P}}^{1,2,+} \varphi(t, \mathbf{x})$ as the set of elements $(r, \mathbf{q}, \mathbf{Q}) \in \mathbb{R} \times$ $\mathbb{R}^{2} \times \mathcal{S}_{2}$ for which there exists a sequence $\left(t_{m}, \mathbf{x}_{m}, r_{m}, \mathbf{q}_{m}, \mathbf{Q}_{m}\right)_{m} \subset \mathbb{R}_{+}^{3} \times$ $\mathcal{P}^{1,2,+} \varphi\left(t_{m}, \mathbf{x}_{m}\right)$ satisfying $\left(t_{m}, \mathbf{x}_{m}, r_{m}, \mathbf{q}_{m}, \mathbf{Q}_{m}\right) \rightarrow(t, \mathbf{x}, r, \mathbf{q}, \mathbf{Q})$. We also define the subjets

$$
\mathcal{P}^{1,2,-} \varphi(t, \mathbf{x}):=\mathcal{P}^{1,2,+}(-\varphi)(t, \mathbf{x}), \quad \overline{\mathcal{P}}^{1,2,-} \varphi(t, \mathbf{x})=-\overline{\mathcal{P}}^{1,2,+}(-\varphi)(t, \mathbf{x}) .
$$

(2) We say that a function $\hat{v} \in C\left(\mathbb{R}_{+}^{3} ; \mathbb{R}\right.$ ) is a viscosity subsolution (resp. 
supersolution) to $(38)$ at $(t, x, y) \in \mathbb{R}_{+}^{3}$ iff

$$
-r+(\beta+\lambda) \hat{v}(t, x, y)-\lambda G[\mathcal{H} \hat{v}](t, x, y)-\sup _{c \geq 0, \pi \in \mathbb{R}} H_{c v}(y, \mathbf{q}, \mathbf{Q} ; c, \pi) \leq 0,
$$

for all $(r, \mathbf{q}, \mathbf{Q}) \in \overline{\mathcal{P}}^{1,2,+} \hat{v}(t, x, y)\left(\right.$ resp. $\left.\geq, \overline{\mathcal{P}}^{1,2,-} \hat{v}(t, x, y)\right)$.

(3) We say that a continuous function $\hat{v} \in C\left(\mathbb{R}_{+}^{3} ; \mathbb{R}\right)$ is a constrained viscosity solution to (38) iff it is a viscosity subsolution on $\mathbb{R}_{+}^{3}$, a viscosity supersolution on $\left.\mathbb{R}_{+} \times\right] 0,+\infty\left[\times \mathbb{R}_{+}\right.$, and satisfies the Dirichlet type boundary condition

$$
\hat{v}(t, 0, y)=\mathbb{E}\left[\int_{t}^{\infty} e^{-(\beta+\lambda)(s-t)} \lambda G[\mathcal{H} \hat{v}]\left(s, 0, Y_{s}^{t, y}\right) d s\right], \quad \forall t \geq 0, \forall y \geq 0
$$

Remark 3.1 The concept of constrained viscosity solution we use naturally comes from the stochastic control problem. The boundaries $\{x=0\}$ and $\{y=$ $0\}$ are both absorbing for the control problem (in the sense that starting from these boundaries, the trajectories of the control problem remain therein), but they have different features. Indeed starting from the boundary $\{y=0\}$ the control problem degenerates into a one dimensional control problem; the associated HJB equation is nothing else than our HJB equation restricted to this boundary

$$
\begin{aligned}
& -\hat{v}_{t}(\cdot, \cdot, 0)+(\beta+\lambda) \hat{v}(\cdot, \cdot, 0)-\lambda G[\mathcal{H} \hat{v}](\cdot, \cdot, 0) \\
& -\sup _{c \geq 0, \pi \in \mathbb{R}}\left\{U(c)+\left(\pi b_{L}-c\right) D_{x} \hat{v}(\cdot, \cdot, 0)+\frac{\sigma_{L}^{2} \pi^{2}}{2} D_{x x} \hat{v}(\cdot, \cdot, 0)\right\}=0,
\end{aligned}
$$

and this is why we require viscosity sub- and supersolution properties at this boundary. Instead, starting from the boundary $\{x=0\}$ there is no control problem (since $\mathcal{A}_{t}(0)=\{(0,0)\}$ ) and the natural condition to impose is a Dirichlet boundary condition.

We can state and prove our main theoretical result.

Theorem 3.1 $\widehat{V}$ is the unique constrained viscosity solution to (38) satisfying the growth condition (26).

Proof. Existence. Once one replaces the term $G[\mathcal{H} \widehat{V}]$ with $G[V]$ in the equation (according to the coupling relation $(15))^{2}$, the fact that $\widehat{V}$ is a viscosity subsolution on the set $\mathbb{R}_{+}^{3}$ and a viscosity supersolution on the set $\left.\mathbb{R}_{+} \times\right] 0,+\infty[\times] 0,+\infty[$ is standard (see, e.g., Ch. 4 in [20] or Ch. V in [21] for the viscosity properties at the interior, and [22] for the viscosity properties at the boundary in the constrained case). The Dirichlet boundary condition (39) is verified due to (15) and (24). The growth condition (26) has been already proved in Proposition 3.4.

\footnotetext{
2 Note that even though the HJB equation is nonlocal, the fact that $\widehat{V}$ is a viscosity solution is standard. This is because the nonlocal term $G[\mathcal{H} \widehat{V}]$ only enters as a running cost in the control problem (there are no jumps involved).
} 
Therefore, it remains to show that $\widehat{V}$ is a supersolution at the boundary $\{y=0\}$. Clearly we have

$$
(p, \mathbf{q}, \mathbf{Q}) \in \mathcal{P}^{1,2,-} \widehat{V}(t, x, 0) \Rightarrow\left(p, q_{1}, Q_{11}\right) \in \mathcal{P}^{1,2,-}(\widehat{V}(\cdot, \cdot, 0))(t, x) .
$$

Since standard arguments apply to the control problem restricted at the boundary $\{y=0\}$, the viscosity supersolution property holds for the function $\widehat{V}(\cdot, \cdot, 0)$ with regard to HJB equation (40) restricted at this boundary. Hence, by (41), we have the supersolution property of $\widehat{V}$ at this boundary for elements of the subjet $\mathcal{P}^{1,2,-} \widehat{V}(t, x, 0)$. To prove the supersolution property for elements of the closure $\overline{\mathcal{P}}^{1,2,-} \widehat{V}(t, x, 0)$, note that if $\left(t_{n}, x_{n}, y_{n}\right) \rightarrow(t, x, 0)$, and $\left(r_{n}, \mathbf{q}_{n}, \mathbf{Q}_{n}\right) \rightarrow(r, \mathbf{q}, \mathbf{Q})$ with $\left(r_{n}, \mathbf{q}_{n}, \mathbf{Q}_{n}\right) \in \mathcal{P}^{1,2,-} \widehat{V}\left(t_{n}, x_{n}, y_{n}\right)$, then for any $c \geq 0, \pi \in \mathbb{R}$, by continuity we have

$$
\begin{aligned}
& -r+(\beta+\lambda) \widehat{V}(t, x, 0)-\lambda G[\mathcal{H} \widehat{V}](t, x, 0)-H_{c v}(0, \mathbf{q}, \mathbf{Q} ; c, \pi) \\
= & \lim _{n \rightarrow \infty}\left(-r_{n}+(\beta+\lambda) \widehat{V}\left(t_{n}, x_{n}, y_{n}\right)-\lambda G[\mathcal{H} \widehat{V}]\left(t_{n}, x_{n}, y_{n}\right)-H_{c v}\left(y_{n}, \mathbf{q}_{n}, \mathbf{Q}_{n} ; c, \pi\right)\right) .
\end{aligned}
$$

Due to the viscosity supersolution property of $\widehat{V}$ in the interior of the state space, and to the viscosity supersolution property at the boundary $\{y=0\}$ for elements of the superjet that we have proved above, we see that the right hand side above is greater than 0 . Hence, taking the infimum over $c \geq 0, \pi \in \mathbb{R}$ shows that

$$
-r+(\beta+\lambda) \widehat{V}(t, x, 0)-\lambda G[\mathcal{H} \widehat{V}](t, x, 0)-\sup _{c \geq 0, \pi \in \mathbb{R}} H_{c v}(0, \mathbf{q}, \mathbf{Q} ; c, \pi) \geq 0
$$

Uniqueness. It is consequence of the comparison principle (Proposition 3.5 below).

Proposition 3.5 (Comparison principle) Let $\hat{w}_{1}$ (resp. $\left.\hat{w}_{2}\right)$ be a viscosity subsolution (resp. supersolution) to (38) on $\left.\mathbb{R}_{+} \times\right] 0,+\infty\left[\times \mathbb{R}_{+}\right.$. Assume that $\hat{w}_{1}, \hat{w}_{2}$ satisfy the growth condition (26), and the boundary condition

$$
\hat{w}_{1}(t, 0, y) \leq \mathbb{E} \int_{t}^{\infty} e^{-(\beta+\lambda)(s-t)} \lambda G\left[\mathcal{H} \hat{w}_{1}\right]\left(s, 0, Y_{s}^{t, y}\right) d s
$$

(resp. $\geq$ for $\left.\hat{w}_{2}\right)$. Then $\hat{w}_{1} \leq \hat{w}_{2}$ on $\mathbb{R}_{+}^{3}$.

Proof. Step 1. Starting from $\hat{w}_{2}$, we construct a sequence of supersolutions $\left(\hat{w}_{2, n}\right)_{n \geq 1}$ that will be used in the next step to show the comparison. Fix some $\left.p^{\prime} \in\right] p, 1[$ such that

$$
\beta \geq k_{p^{\prime}}=\frac{p^{\prime}}{1-p^{\prime}} \frac{b_{L}^{2}}{2 \sigma_{L}^{2}}+\tilde{k}_{p^{\prime}}
$$

Finding such a $p^{\prime}$ is possible by (3) and by the fact that $p^{\prime} \mapsto k_{p^{\prime}}$ is continuous. Define

$$
f^{p^{\prime}}(t, x, y):=e^{\tilde{k}_{p^{\prime}} t}(x+y)^{p^{\prime}}
$$


We claim that on $\left.\mathbb{R}_{+} \times\right] 0,+\infty\left[\times \mathbb{R}_{+}\right.$

$$
-f_{t}^{p^{\prime}}+(\beta+\lambda) f^{p^{\prime}}-\lambda G\left[\mathcal{H} f^{p^{\prime}}\right]-\sup _{\pi \in \mathbb{R}} H_{c v}\left(y, D_{(x, y)} f^{p^{\prime}}, D_{(x, y)}^{2} f^{p^{\prime}} ; 0, \pi\right) \geq 0
$$

Indeed, first we observe that $G\left[\mathcal{H} f^{p^{\prime}}\right] \leq f^{p^{\prime}}$ by Proposition $3.3(\mathrm{v})$, and then by straightforward computations we can check that

$$
\begin{aligned}
\sup _{\pi \in \mathbb{R}}\left[\pi b_{L} f_{x}^{p^{\prime}}+\frac{\rho b_{L} \sigma_{I}}{\sigma_{L}} y f_{y}^{p^{\prime}}+\frac{\sigma_{L}^{2} \pi^{2}}{2} f_{x x}^{p^{\prime}}+\pi \rho \sigma_{I} \sigma_{L} y f_{x y}^{p^{\prime}}+\rho^{2} \frac{\sigma_{I}^{2}}{2} y^{2} \hat{f}_{y y}^{p^{\prime}}\right] \\
=\frac{p^{\prime}}{1-p^{\prime}} \frac{b_{L}^{2}}{2 \sigma_{L}^{2}} f^{p^{\prime}}
\end{aligned}
$$

Hence, using (43) we obtain (44). Now given an integer $n \geq 1$, consider the function

$$
\hat{w}_{2, n}:=\hat{w}_{2}+\frac{1}{n} f^{p^{\prime}}
$$

We claim that for any $\left.(t, x, y) \in \mathbb{R}_{+} \times\right] 0,+\infty\left[\times \mathbb{R}_{+}\right.$, the function $\hat{w}_{2, n}$ is a supersolution to $(38)$ at $(t, x, y)$. Indeed, notice that

$$
\mathcal{P}^{1,2,-} \hat{w}_{2, n}(t, x, y)=\mathcal{P}^{1,2,-} \hat{w}_{2}(t, x, y)+\frac{1}{n}\left(f_{t}^{p^{\prime}}, D_{(x, y)} f^{p^{\prime}}, D_{(x, y)}^{2} f^{p^{\prime}}\right)(t, x, y)
$$

So, using subadditivity of $\mathcal{H}$, linearity of $G$, the fact that $f_{x}^{p^{\prime}} \geq 0$, linearity of $H_{c v}$ in $(\mathbf{q}, \mathbf{Q})$ and $(44)$, we have for all $(r, \mathbf{q}, \mathbf{Q}) \in \mathcal{P}^{1,2,-} \hat{w}_{2}(t, x, y)$

$$
\begin{aligned}
& -\left(r+\frac{1}{n} f_{t}^{p^{\prime}}(t, x, y)\right)+(\beta+\lambda)\left(\hat{w}_{2}(t, x, y)\right. \\
+ & \left.\frac{1}{n} f^{p^{\prime}}(t, x, y)\right)-\lambda G\left[\mathcal{H}\left(\hat{w}_{2}+\frac{1}{n} f^{p^{\prime}}\right)\right](t, x, y) \\
- & \sup _{c \geq 0, \pi \in \mathbb{R}} H_{c v}\left(y, \mathbf{q}+\frac{1}{n} D_{(x, y)} f^{p^{\prime}}(t, x, y), \mathbf{Q}+\frac{1}{n} D_{(x, y)}^{2} f^{p^{\prime}}(t, x, y) ; c, \pi\right) \\
\geq & -r+(\beta+\lambda) \hat{w}_{2}(t, x, y)-\lambda G\left[\mathcal{H} \hat{w}_{2}\right](t, x, y)-\sup _{c \geq 0, \pi \in \mathbb{R}} H_{c v}(y, \mathbf{q}, \mathbf{Q} ; c, \pi) \\
+ & \frac{1}{n}\left\{-f_{t}^{p^{\prime}}(t, x, y)+(\beta+\lambda) f^{p^{\prime}}(t, x, y)-\lambda G\left[\mathcal{H} f^{p^{\prime}}\right](t, x, y)\right. \\
& \left.-\sup _{\pi \in \mathbb{R}} H_{c v}\left(y, D_{(x, y)} f^{p^{\prime}}(t, x, y), D_{(x, y)}^{2} f^{p^{\prime}}(t, x, y) ; 0, \pi\right)\right\} \geq 0 .
\end{aligned}
$$


This shows that actually $\hat{w}_{2, n}$ is a supersolution to $(38)$ at $(t, x, y)$ for each $n \geq 1$. Moreover,

$$
\begin{aligned}
& \lambda \mathbb{E} \int_{t}^{\infty} e^{-(\beta+\lambda)(s-t)} G\left[\mathcal{H} f^{p^{\prime}}\right]\left(s, 0, Y_{s}^{t, y}\right) d s \\
\leq & e^{\tilde{k}_{p^{\prime}} t} y^{p^{\prime}} \lambda \mathbb{E} \int_{t}^{\infty} e^{\left(-\beta-\lambda+\tilde{k}_{p^{\prime}}\right)(s-t)}\left(Y_{s}^{t, 1}\right)^{p^{\prime}} d s \\
\leq & f^{p^{\prime}}(t, 0, y) \lambda \int_{t}^{\infty} e^{\left(-\beta-\lambda+k_{p^{\prime}}\right)(s-t)} d s \\
= & \frac{\lambda}{\beta-k_{p^{\prime}}+\lambda} f^{p^{\prime}}(t, 0, y) \\
\leq & f^{p^{\prime}}(t, 0, y),
\end{aligned}
$$

where in the second inequality we have used (22). By subadditivity of $\mathcal{H}$ and linearity of $G$, it follows that

$$
\hat{w}_{2, n}(t, 0, y) \leq \mathbb{E} \int_{t}^{\infty} e^{-(\beta+\lambda)(s-t)} \lambda G\left[\mathcal{H} \hat{w}_{2, n}\right]\left(s, 0, Y_{s}^{t, y}\right) d s .
$$

Finally, notice that by the growth condition on $\hat{w}_{1}$ and $\hat{w}_{2}$ we have

$$
\lim _{|(t, x, y)| \rightarrow \infty}\left(\hat{w}_{1}-\hat{w}_{2, n}\right)(t, x, y)=-\infty .
$$

Step 2. We show that for all $n \geq 1$, it is $\hat{w}_{1} \leq \hat{w}_{2, n}$ on $\mathbb{R}_{+}^{3}$, and thus conclude that $\hat{w}_{1} \leq \hat{w}_{2}$. Fix $n \geq 1$ and define

$$
M:=\sup _{[0,+\infty) \times \mathbb{R}_{+}^{2}}\left(\hat{w}_{1}-\hat{w}_{2, n}\right) .
$$

We want to show that $M \leq 0$. By (46) and continuity of $\hat{w}_{1}, \hat{w}_{2, n}$, we see that, for some $T_{0}>0$, for some compact set $\mathcal{C} \subset \mathbb{R}_{+}^{2}$, and some $(\bar{t}, \bar{x}, \bar{y}) \in\left[0, T_{0}\right] \times \mathcal{C}$,

$$
M=\max _{\left[0, T_{0}\right] \times \mathcal{C}^{2}}\left(\hat{w}_{1}-\hat{w}_{2, n}\right)=\left(\hat{w}_{1}-\hat{w}_{2, n}\right)(\bar{t}, \bar{x}, \bar{y}) .
$$

We now distinguish between two cases, showing that both of them lead to conclude $M \leq 0$.

Case 1: $\bar{x}=0$. First note that $\mathcal{H} \hat{w}_{1}-\mathcal{H} \hat{w}_{2, n} \leq M$. Using the boundary condition (42), we then have

$$
\begin{aligned}
M & =\left(\hat{w}_{1}-\hat{w}_{2, n}\right)(\bar{t}, 0, \bar{y}) \\
& \leq \mathbb{E} \int_{\bar{t}}^{\infty} e^{-(\beta+\lambda)(s-t)} \lambda G\left[\mathcal{H} \hat{w}_{1}-\mathcal{H} \hat{w}_{2, n}\right]\left(s, 0, Y_{s}^{\bar{t}, \bar{y}}\right) d s \\
& \leq \int_{\bar{t}}^{\infty} e^{-(\beta+\lambda)(s-t)} \lambda M d s \\
& =\frac{\lambda}{\beta+\lambda} M,
\end{aligned}
$$


and it follows that $M \leq 0$.

Case 2: $\bar{x}>0$. Using viscosity properties of $\hat{w}_{1}$ and $\hat{w}_{2, n}$, the nonnegativity of an interior maximum may be proved by the "doubling of variables" technique as in $[23]$. Let $\varepsilon>0$, and define on $\left[0, T_{0}\right] \times \mathcal{C}^{2}$ the function

$$
\Phi_{\varepsilon}\left(t, \mathbf{x}, \mathbf{x}^{\prime}\right)=\hat{w}_{1}(t, \mathbf{x})-\hat{w}_{2, n}\left(t, \mathbf{x}^{\prime}\right)-\frac{\left|\mathbf{x}-\mathbf{x}^{\prime}\right|^{2}}{2 \varepsilon}, \quad \mathbf{x}=(x, y), \quad \mathbf{x}^{\prime}=\left(x^{\prime}, y^{\prime}\right)
$$

Since $\Phi_{\varepsilon}$ is continuous on the compact set $\left[0, T_{0}\right] \times \mathcal{C}^{2}$, there exists $\left(t_{\varepsilon}, \mathbf{x}_{\varepsilon}, \mathbf{x}_{\varepsilon}^{\prime}\right)$ such that

$$
M_{\varepsilon}:=\sup _{\left[0, T_{0}\right] \times(\mathcal{C})^{2}} \Phi_{\varepsilon}=\Phi_{\varepsilon}\left(t_{\varepsilon}, \mathbf{x}_{\varepsilon}, \mathbf{x}_{\varepsilon}^{\prime}\right)
$$

Letting $\varepsilon \rightarrow 0$, there exists a sequence, still denoted (with a slight abuse of notation) by $\left(t_{\varepsilon}, \mathbf{x}_{\varepsilon}, \mathbf{x}_{\varepsilon}^{\prime}\right)$, converging to some $\left(\hat{t}, \hat{\mathbf{x}}, \hat{\mathbf{x}}^{\prime}\right)$. By standard arguments (see e.g. Lemma 3.1 in [23]), we have

$$
\lim _{\varepsilon \rightarrow 0} \frac{\left|\mathbf{x}_{\varepsilon}-\mathbf{x}_{\varepsilon}^{\prime}\right|^{2}}{2 \varepsilon}=0
$$

so it follows that it must be $\hat{\mathbf{x}}=\hat{\mathbf{x}}^{\prime}$ and consequently that $(\hat{t}, \hat{\mathbf{x}})$ is a maximum point of $\hat{w}_{1}-\hat{w}_{2, n}$. Hence, without any loss of generality we can take in (47)

$$
(\bar{t}, \bar{x}, \bar{y})=(\hat{t}, \hat{\mathbf{x}}) .
$$

Now we apply the parabolic Ishii lemma (Th. 8.3 in [23]) to obtain $r, r^{\prime} \in \mathbb{R}$, $\mathbf{Q}, \mathbf{Q}^{\prime}$ in $\mathcal{S}_{2}$ such that

$$
\begin{aligned}
\left(r, \frac{\mathbf{x}_{\varepsilon}-\mathbf{x}_{\varepsilon}^{\prime}}{\varepsilon}, \mathbf{Q}\right) & \in \overline{\mathcal{P}}^{1,2,+} \hat{w}_{1}\left(t_{\varepsilon}, \mathbf{x}_{\varepsilon}\right), \\
\left(r^{\prime}, \frac{\mathbf{x}_{\varepsilon}-\mathbf{x}_{\varepsilon}^{\prime}}{\varepsilon}, \mathbf{Q}^{\prime}\right) & \in \overline{\mathcal{P}}^{1,2,-} \hat{w}_{2, n}\left(t_{\varepsilon}, \mathbf{q}_{\varepsilon}^{\prime}\right), \\
\left(\begin{array}{cc}
\mathbf{Q} & \mathbf{0} \\
\mathbf{0}-\mathbf{Q}^{\prime}
\end{array}\right) & \leq \frac{3}{\varepsilon}\left(\begin{array}{cc}
\mathbf{I}_{2} & -\mathbf{I}_{2} \\
-\mathbf{I}_{2} & \mathbf{I}_{2}
\end{array}\right), \\
r+r^{\prime} & =0,
\end{aligned}
$$

where we have denoted by $\mathbf{I}_{2}$ the identity matrix of $\mathbb{R}^{2 \times 2}$. Since $\mathbf{x}_{\varepsilon}=\left(x_{\varepsilon}, y_{\varepsilon}\right)$ converges to $\hat{\mathbf{x}}=(x, y)$, we have $x_{\varepsilon}>0$ for $\varepsilon$ small enough, and we can use the viscosity subsolution property of $\hat{w}_{1}$ to obtain

$$
\begin{array}{r}
-r+(\beta+\lambda) \hat{w}_{1}\left(t_{\varepsilon}, x_{\varepsilon}, y_{\varepsilon}\right)-\widetilde{U}\left(\frac{x_{\varepsilon}-x_{\varepsilon}^{\prime}}{\varepsilon}\right)-\lambda G\left[\mathcal{H} \hat{w}_{1}\right]\left(t_{\varepsilon}, x_{\varepsilon}, y_{\varepsilon}\right) \\
-\sup _{\pi \in \mathbb{R}}\left[\pi \frac{x_{\varepsilon}-x_{\varepsilon}^{\prime}}{\varepsilon}+\frac{\rho b_{L} \sigma_{I}}{\sigma_{L}} y_{\varepsilon} \frac{y_{\varepsilon}-y_{\varepsilon}^{\prime}}{\varepsilon}\right. \\
\left.+\frac{\sigma_{L}^{2} \pi^{2}}{2} Q_{11}+\pi \rho \sigma_{I} \sigma_{L} y_{\varepsilon} Q_{12}+\rho^{2} \frac{\sigma_{I}^{2}}{2} y_{\varepsilon}^{2} Q_{22}\right] \leq 0
\end{array}
$$


and the supersolution property of $\hat{w}_{2, n}$ to get

$$
\begin{aligned}
& -r^{\prime}+(\beta+\lambda) \hat{w}_{2, n}\left(t_{\varepsilon}, x_{\varepsilon}^{\prime}, y_{\varepsilon}^{\prime}\right)-\widetilde{U}\left(\frac{x_{\varepsilon}-x_{\varepsilon}^{\prime}}{\varepsilon}\right)-\lambda G\left[\mathcal{H} \hat{w}_{2, n}\right]\left(t_{\varepsilon}, x_{\varepsilon}^{\prime}, y_{\varepsilon}^{\prime}\right) \\
& -\sup _{\pi \in \mathbb{R}}\left[\pi \frac{x_{\varepsilon}-x_{\varepsilon}^{\prime}}{\varepsilon}+\frac{\rho b_{L} \sigma_{I}}{\sigma_{L}} y_{\varepsilon}^{\prime} \frac{y_{\varepsilon}-y_{\varepsilon}^{\prime}}{\varepsilon}\right. \\
& \left.+\frac{\sigma_{L}^{2} \pi^{2}}{2} Q_{11}^{\prime}+\pi \rho \sigma_{I} \sigma_{L} y_{\varepsilon}^{\prime} Q_{12}^{\prime}+\rho^{2} \frac{\sigma_{I}^{2}}{2}\left(y_{\varepsilon}^{\prime}\right)^{2} Q_{22}^{\prime}\right] \geq 0 .
\end{aligned}
$$

Subtracting (53) from (54), using the fact that the difference of the supremum is less than the supremum of the difference and (52), we obtain

$$
\begin{aligned}
& (\beta+\lambda)\left(\hat{w}_{1}\left(t_{\varepsilon}, x_{\varepsilon}, y_{\varepsilon}\right)-\hat{w}_{2, n}\left(t_{\varepsilon}, x_{\varepsilon}^{\prime}, y_{\varepsilon}^{\prime}\right)\right) \\
\leq & \sup _{\pi \in \mathbb{R}}\left[\frac{\sigma_{L}^{2} \pi^{2}}{2}\left(Q_{11}-Q_{11}^{\prime}\right)+\pi \rho \sigma_{I} \sigma_{L}\left(y_{\varepsilon} Q_{12}-y_{\varepsilon}^{\prime} Q_{12}^{\prime}\right)+\rho^{2} \frac{\sigma_{I}^{2}}{2}\left(y_{\varepsilon}^{2} Q_{22}-\left(y_{\varepsilon}^{\prime}\right)^{2} Q_{22}^{\prime}\right)\right] \\
& +\frac{\rho b_{L} \sigma_{I}}{\sigma_{L}} \frac{\left(y_{\varepsilon}-y_{\varepsilon}^{\prime}\right)^{2}}{\varepsilon}+\lambda\left(G\left[\mathcal{H} \hat{w}_{1}\right]\left(t_{\varepsilon}, x_{\varepsilon}, y_{\varepsilon}\right)-G\left[\mathcal{H} \hat{w}_{2, n}\right]\left(t_{\varepsilon}, x_{\varepsilon}^{\prime}, y_{\varepsilon}^{\prime}\right)\right) .
\end{aligned}
$$

Now notice that

$$
\begin{aligned}
\lim _{\varepsilon \rightarrow 0}\left(G\left[\mathcal{H} \hat{w}_{1}\right]\left(t_{\varepsilon}, x_{\varepsilon}, y_{\varepsilon}\right)-G\left[\mathcal{H} \hat{w}_{2, n}\right]\left(t_{\varepsilon}, x_{\varepsilon}^{\prime}, y_{\varepsilon}^{\prime}\right)\right) & =G\left[\mathcal{H} \hat{w}_{1}\right](\bar{t}, \bar{x}, \bar{y})-G\left[\mathcal{H} \hat{w}_{2, n}\right](\bar{t}, \bar{x}, \bar{y}) \\
& \leq \sup _{\mathbb{R}_{+}}\left(\mathcal{H} \hat{w}_{1}-\mathcal{H} \hat{w}_{2, n}\right) \leq M
\end{aligned}
$$

Furthermore, using (51) we see that for all $\pi \in \mathbb{R}$

$$
\begin{aligned}
& \frac{\sigma_{L}^{2} \pi^{2}}{2}\left(Q_{11}-Q_{11}^{\prime}\right)+\pi \rho \sigma_{I} \sigma_{L}\left(y_{\varepsilon} Q_{12}-y_{\varepsilon}^{\prime} Q_{12}^{\prime}\right)+\rho^{2} \frac{\sigma_{I}^{2}}{2}\left(y_{\varepsilon}^{2} Q_{22}-\left(y_{\varepsilon}^{\prime}\right)^{2} Q_{22}^{\prime}\right) \\
= & \frac{1}{2}\left(\sigma_{L} \pi \rho \sigma_{I} y_{\varepsilon} \sigma_{L} \pi \rho \sigma_{I} y_{\varepsilon}^{\prime}\right)\left(\begin{array}{cc}
\mathbf{Q} & \mathbf{0} \\
\mathbf{0} & -\mathbf{Q}^{\prime}
\end{array}\right)\left(\begin{array}{c}
\sigma_{L} \pi \\
\rho \sigma_{I} y_{\varepsilon} \\
\sigma_{L} \pi \\
\rho \sigma_{I} y_{\varepsilon}^{\prime}
\end{array}\right) \\
\leq & \frac{1}{2}\left(\sigma_{L} \pi \rho \sigma_{I} y_{\varepsilon} \sigma_{L} \pi \rho \sigma_{I} y_{\varepsilon}^{\prime}\right)\left(\begin{array}{cc}
\mathbf{I}_{2} & -\mathbf{I}_{2} \\
-\mathbf{I}_{2} & \mathbf{I}_{2}
\end{array}\right)\left(\begin{array}{c}
\sigma_{L} \pi \\
\rho \sigma_{I} y_{\varepsilon} \\
\sigma_{L} \pi \\
\rho \sigma_{I} y_{\varepsilon}^{\prime}
\end{array}\right) \\
\leq & \left(\rho \sigma_{I}\right)^{2} \frac{3}{2 \varepsilon}\left|y_{\varepsilon}-y_{\varepsilon}^{\prime}\right|^{2} .
\end{aligned}
$$

Recall that by (48)

$$
\frac{\left(y_{\varepsilon}-y_{\varepsilon}^{\prime}\right)^{2}}{\varepsilon} \rightarrow 0 \quad \text { when } \varepsilon \rightarrow 0 .
$$

So, letting $\varepsilon$ go to 0 in (55), and combining (56)-(57)-(58), we finally obtain

$$
(\beta+\lambda) M \leq \lambda M,
$$

so $M \leq 0$. 


\section{An Iterative Approximation Scheme for the Value Functions}

In this section we present an iterative scheme to compute numerical approximations of the value functions $V$ and $\widehat{V}$. For sake of brevity we omit the proofs of the results that can be found in [24].

First of all, we observe that (38) contains a nonlocal term, i.e. $G[\mathcal{H} \widehat{V}]$. Thus, in order to get a computational tool to approximate $V$ and $\widehat{V}$, it is needed to couple standard numerical schemes with an iterative procedure as we are going to describe.

We start with

$$
V^{0}=0
$$

Then, inductively:

- Given $n \geq 0$ and $V^{n}$, we define $\widehat{V}^{n}$ on $\mathbb{R}_{+}^{3}$ as the unique (constrained viscosity) solution to

$$
\begin{aligned}
-\widehat{V}_{t}^{n}+(\beta+\lambda) \widehat{V}^{n}-\lambda G\left[V^{n}\right] & \\
& -\sup _{c \geq 0, \pi \in \mathbb{R}} H_{c v}\left(y, D_{(x, y)} \widehat{V}^{n}, D_{(x, y)}^{2}, \widehat{V}^{n} ; c, \pi\right)=0,
\end{aligned}
$$

with boundary condition

$$
\widehat{V}^{n}(t, 0, y)=\mathbb{E} \int_{t}^{\infty} e^{-(\beta+\lambda)(s-t)} \lambda G\left[V^{n}\right]\left(s, 0, \tilde{Y}_{s}^{t, y}\right) d s .
$$

and growth condition

$$
\left|\widehat{V}^{n}(t, x, y)\right| \leq K e^{\tilde{k}_{p} t}(x+y)^{p} .
$$

- Given $n \geq 0$ and $\widehat{V}^{n}$, we define $V^{n+1}$ by

$$
V^{n+1}=\mathcal{H} \widehat{V}^{n}
$$

The next result states the convergence of $V^{n}$ to $V$ and of $\widehat{V}^{n}$ to $\widehat{V}$ at an exponential rate.

Proposition 4.1 For some $K>0$, we have

$$
\begin{aligned}
0 & \leq\left(V-V^{n}\right)(r) \leq K r^{p} \delta^{n}, \\
0 \leq\left(\widehat{V}-\widehat{V}^{n}\right)(t, x, y) & \leq K e^{\tilde{k}_{p} t}(x+y)^{p} \delta^{n},
\end{aligned}
$$

where

$$
\delta:=\frac{\lambda}{\lambda+\beta-k_{p}}<1
$$

To solve the PDE (60) one needs to approximate it by a finite horizon PDE. To this end, we fix some finite horizon $T>0$ and consider the functions $\widehat{V}^{n, T}$, $V^{n, T}$ defined recursively as follows: 
- $V^{0, T}=0$.

- Given $n \geq 0$ and $V^{n, T}$, and given some terminal boundary condition condition $\phi^{n, T}$, we define on $[0, T] \times \mathbb{R}_{+}^{2}$

$$
\begin{aligned}
& \widehat{V}^{n, T}(t, x, y) \\
&=\sup _{(c, \pi) \in \mathcal{A}_{t}(x)} \mathbb{E}\left[\int_{t}^{T} e^{-(\beta+\lambda)(s-t)}\left(U\left(c_{s}\right)+\lambda G\left[V^{n, T}\right]\left(s, \tilde{X}_{s}^{t, x, \pi, c}, \tilde{Y}_{s}^{t, y}\right)\right) d s\right. \\
&\left.+e^{-(\beta+\lambda)(T-t)} \phi^{n, T}\left(\tilde{X}_{s}^{t, x, \pi, c}, \tilde{Y}_{s}^{t, y}\right)\right],
\end{aligned}
$$

- Given $n \geq 0$ and $\widehat{V}^{n, T}$ we define

$$
V^{n+1, T}=\mathcal{H} \widehat{V}^{n, T}
$$

By the same methods as above it is then straightforward to check that, for each $n \geq 0, \widehat{V}^{n, T}$ is a constrained viscosity solution on $\left[0, T\left[\times \mathbb{R}_{+}^{2}\right.\right.$ to

$$
\begin{aligned}
-\widehat{V}_{t}^{n, T}+(\beta+\lambda) \widehat{V}^{n, T}-\lambda G\left[V^{n, T}\right](t, x, y) & \\
& -\sup _{c \geq 0, \pi \in \mathbb{R}} H_{c v}\left(y, D_{(x, y)} \widehat{V}^{n, T}, D_{(x, y)}^{2} \widehat{V}^{n, T} ; c, \pi\right)=0,
\end{aligned}
$$

with boundary conditions

$$
\widehat{V}^{n, T}(T, x, y)=\phi^{n, T}(x, y),
$$

and

$$
\begin{aligned}
& \widehat{V}^{n, T}(t, 0, y) \\
= & \mathbb{E}\left[\int_{t}^{T} e^{-(\beta+\lambda)(s-t)} \lambda G\left[V^{n, T}\right]\left(s, 0, \tilde{Y}_{s}^{t, y}\right) d s+e^{-(\beta+\lambda)(T-t)} \phi^{n, T}\left(0, \tilde{Y}_{s}^{t, x, \pi, c}\right)\right] .
\end{aligned}
$$

Now we assume that the terminal condition $\phi^{n, T}$ satisfies

$$
\left|\phi^{n, T}(x, y)-\widehat{V}^{n}(T, x, y)\right| \leq \mathcal{E} e^{\tilde{k}_{p} T}(x+y)^{p},
$$

for some error $\mathcal{E}$ not depending on $n$. Note that this assumption is not restrictive since $0 \leq \widehat{V}^{n} \leq \widehat{V}$, and so due to (26), the inequality (67) is satisfied, e.g., by taking $\phi^{n, T}=0$.

We then have the following estimate for the numerical error induced by the finite horizon approximation:

Proposition 4.2 For every $n \geq 1$ and every $t \in[0, T], r, x, y \in \mathbb{R}_{+}$,

$$
\begin{aligned}
\left|\left(V^{n, T}-V^{n}\right)(r)\right| & \leq \frac{\mathcal{E}}{1-\delta} e^{-\left(\beta+\lambda-k_{p}\right) T} r^{p}, \\
\left|\left(\widehat{V}^{n, T}-\widehat{V}^{n}\right)(t, x, y)\right| & \leq \frac{\mathcal{E}}{1-\delta} e^{-\left(\beta+\lambda-k_{p}\right) T} e^{\tilde{k}_{p} t}(x+y)^{p} .
\end{aligned}
$$


By combining Propositions 4.1 and 4.2, one can choose $n, T$ large enough to approximate $V, \widehat{V}$ by $V^{n, T}, \widehat{V}^{n, T}$ (respectively) with any required precision. The latter ones can be computed by the iterative procedure described above, using at each step of the iteration a standard explicit finite-difference scheme for parabolic viscosity solutions to solve the PDE (see, e.g., Ch. IX in [21] for a description of the scheme, as well as the proof of its convergence). Finally, we observe that the choice of $n$ and $T$ has to depend on $\lambda$ :

- When $\lambda$ is large, $\delta$ is close to 1 so that the number of iterations $n$ must be chosen large.

- The finite horizon error is roughly speaking of order $(1+\lambda) e^{-(1+\lambda) T}$, so that $T$ may be chosen small for large $\lambda$ and must be reasonably large for small $\lambda$.

\section{Cost of Illiquidity and Optimal Policy in the Illiquid Asset}

The results obtained allow us to measure the cost of illiquidity and to determine the optimal policy allocation in the illiquid asset. Indeed, $\widehat{V}$ can be computed numerically following the scheme described in Section 4, and then the optimal allocation $\left(\alpha_{k}^{*}\right)_{k \geq 0}$ in the illiquid asset and the value function $V$ can be derived.

At $\tau_{0}=0$ the optimal allocation in the illiquid asset is

$$
\alpha_{0}^{*}=\operatorname{argmax}_{0 \leq a \leq r} \widehat{V}(0, r-a, a) .
$$

and consequently the value function $V$ can be computed.

Figure 1 shows the impact of illiquidity in the case of a power utility function $U(c)=c^{p} / p$. In this case, by standard arguments using the homogeneity of $U$, one can prove that the value function has the structure $V(r)=V(1) r^{p}$. The value $V(1)$ is represented in Figure 1 as function of $\rho$ for different values of the liquidity parameter $\lambda$. The lines corresponding to the constrained and unconstrained Merton refer to the problem when the asset $I$ is considered as liquid and when, respectively, the constraint $\pi_{I} \in[0,1]$ is imposed or not. The parameters are set as follows:

$$
\beta=0.2, \quad p=0.5, \quad b_{L}=0.15, \quad \sigma_{L}=1, \quad b_{I}=0.2, \quad \sigma_{I}=1 .
$$

We observe, as expected, a monotone convergence to the constrained Merton problem (see also [14] for comments). The difference between the different values of $\lambda$ can be taken as an absolute measure of the cost of illiquidity.

In Figure 2 we plot the optimal investment proportion in the illiquid asset $\alpha_{0}^{*} / r$ as a function of the correlation $\rho$, for some values of the liquidity parameter $\lambda$. Also in this case we observe the monotone convergence to the constrained Merton problem. 


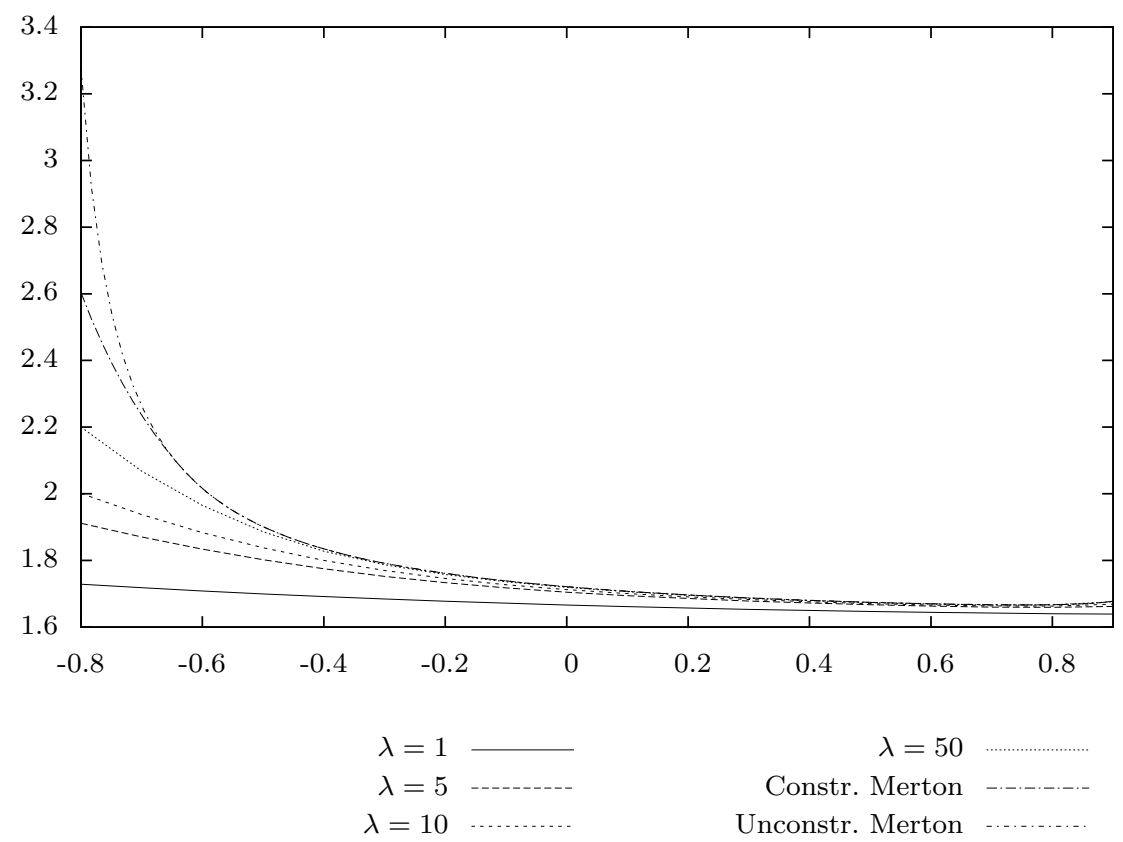

Fig. 1 Value function $V(1)$ as a function of $\rho$ for some values of the liquidity parameter $\lambda$.

\section{Conclusions}

We have investigated a problem of optimal investment-consumption within a market composed by a liquid and an illiquid asset. The problem has been set as a stochastic control problem, which is a mixed continuous/discrete control problem, due to the presence of the illiquid asset. We have rewritten the control problem in a suitable form reducing it to a more standard continuous control problem in order to apply the usual machinery of Dynamic Programming. We have investigated the associated HJB equation by means of viscosity solutions, giving a characterization of the value function as unique viscosity solution of the HJB equation. This has allowed to use a numerical procedure to compute the value function and see, numerically, what is the impact of the illiquidity on the optimal value of the problem, and what is the optimal allocation policy in the illiquid asset.

The study leaves open other issues, such as the optimal allocation policy in the liquid asset and the optimal consumption policy. A deeper study on the regularity of the value function is needed for that, and it is performed, in the special case of power utility, in the companion paper [14] (see also [15]).

Acknowledgements The authors would like to thank two anonymous referees, who with their valuable comments have led to a significant improvement of the paper. Also they are 


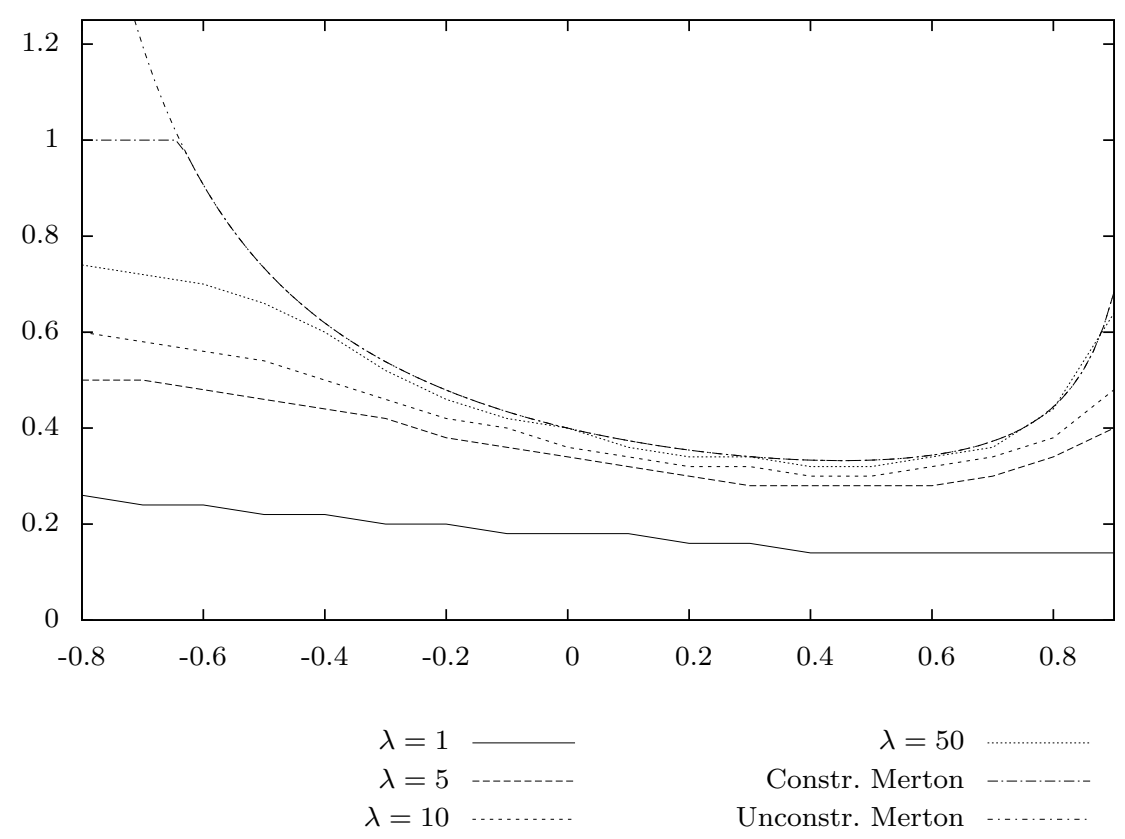

Fig. 2 Optimal investment proportion $\frac{\alpha_{0}^{*}}{r}$ in the illiquid asset as function of $\rho$ for some values of the liquidity parameter $\lambda$.

grateful to Huyên Pham to have suggested to them the topic and to Fausto Gozzi for useful suggestions.

\section{References}

1. Matsumoto K.,Optimal portfolio of low liquid assets with a log-utility function, Fin. Stoch., Vol. 10, pp. 121-145 (2006)

2. Pham H. and P. Tankov, A model of optimal consumption under liquidity risk with random trading times, Math. Fin., Vol. 18, pp. 613-627 (2008).

3. Rogers C. and O. Zane, A simple model of liquidity effects, in Advances in Finance and Stochastics: Essays in Honour of Dieter Sondermann, eds. K. Sandmann and P. Schoenbucher, pp. 161-176 (2002).

4. Kabanov Y., and M. Safarian, Markets with Transaction Costs: Mathematical Theory", Springer-Verlag (2009)

5. Jarrow, R. A.,Derivative Security Markets, Market Manipulation, and Option Pricing Theory, J. Financial Quantitative Anal., Vol. 29, 241-261 (1994)

6. Frey, R., Perfect Option Hedging for a Large Trader, Fin. Stoch. Vol. 2, pp. 115-141 (1998)

7. Frey, R. and A. Stremme, Market Volatility and Feedback Effects from Dynamic Hedging, Math. Fin., Vol. 7, pp. 351-374 (1997)

8. Platen, E., and M. Schweizer, On Feedback Effects from Hedging Derivatives, Math. Fin., Vol. 8, pp. 67-84 (1998).

9. Jouini, E.: Price functionals with bid-ask spreads: an axiomatic approach. J. Math. Econ., Vol. 34, pp. 547-558 (2000). 
10. Cetin U., Jarrow A., and P. Protter, Liquidity risk and arbitrage pricing theory, Fin. Stoch., Vol. 8, pp. 311-341 (2004)

11. Cetin U., Soner H. M., and N. Touzi, Option hedging for small investors under liquidity costs, Fin. Stoch., Vol. 14, pp. 317-341 (2010)

12. Ang A., Papanikolaou D., and M. Westerfield, Portfolio Choice with Illiquid Assets, Preprint (2011)

13. Schwartz E. and C. Tebaldi, Illiquid assets and optimal portfolio choice, NBER Working Paper No. w12633 (2006).

14. Federico S., Gassiat P., and F. Gozzi, Impact of time illiquidity in a mixed market without full observation. Arxiv Preprint (2012)

15. Federico S., Gassiat P., and F. Gozzi, Utility maximization with current utility on the wealth: regularity of solutions to the HJB equation. Arxiv Preprint (2012)

16. Øksendal B. and A. Sulem, Applied stochastic control of jump diffusions, Third edition, Springer-Verlag (2009)

17. Cretarola A., Gozzi F., Pham H. and P. Tankov, Optimal consumption policies in illiquid markets, Fin. Stoch., Vol. 15, pp. 85-115 (2011)

18. Pham H. and P. Tankov, A coupled system of integrodifferential equations arising in liquidity risk mode, App. Math. and Optim., Vol. 59, pp. 147-173 (2009).

19. Pham H., Stochastic control under progressive enlargment of filtrations and applications to multiple defaults risk management, Stoch. Proc. and their Appl., 120, 1795-1820 (2010)

20. Yong J. and X.Y. Zhou, Stochastic Controls: Hamiltonian Systems and HJB Equations, Springer Verlag, New York (1999).

21. Fleming W. H. and H. M. Soner, Controlled Markov Processes and Viscosity Solutions, Springer-Verlag (2006)

22. Ishii H. and P. Loreti : On stochastic optimal control problems with state constraint, Indiana Univ. Math. J., Vol. 51, pp. 1167-1196 (2002)

23. Crandall M., Ishii H., and P.L. Lions, User's Guide to Viscosity Solutions of Second Order Partial Differential Equations, Bull. Amer. Math. Soc., Vol. 27, pp. 1-67, 1992

24. Gassiat P.,Modélisation du risque de liquidité et méthodes de quantification appliquées au contrôle stochastique séquentiel, Phd thesis of University Paris Diderot, available at http://tel.archives-ouvertes.fr/tel-00651357/fr/ (2011)

25. Gassiat P., Gozzi F., and H. Pham, Investment/consumption problem in illiquid markets with regimes switching, Submitted, preprint available at http://www.proba.jussieu.fr/mathdoc/preprints/index.html\#2011 (2012) 